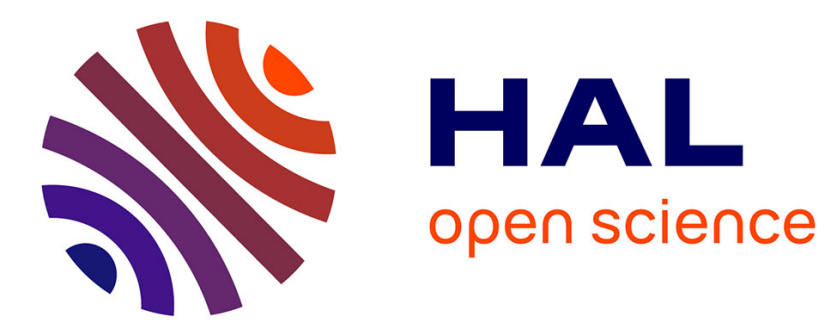

\title{
Study of the brightness of trumpet tones
}

Emilie Poirson, Joël Gilbert, Jean-François Petiot

\section{To cite this version:}

Emilie Poirson, Joël Gilbert, Jean-François Petiot. Study of the brightness of trumpet tones. Journal of the Acoustical Society of America, 2005, 118 (4), pp.2656-2666. hal-00474663

\section{HAL Id: hal-00474663 https://hal.science/hal-00474663}

Submitted on 21 Apr 2010

HAL is a multi-disciplinary open access archive for the deposit and dissemination of scientific research documents, whether they are published or not. The documents may come from teaching and research institutions in France or abroad, or from public or private research centers.
L'archive ouverte pluridisciplinaire HAL, est destinée au dépôt et à la diffusion de documents scientifiques de niveau recherche, publiés ou non, émanant des établissements d'enseignement et de recherche français ou étrangers, des laboratoires publics ou privés. 


\section{Study of the brightness of trumpet tones}

\section{Emilie Poirson $^{1}$, Jean-François Petiot ${ }^{1}$, Joël Gilbert ${ }^{2}$}

${ }^{1}$ Institut de Recherche en Communication et Cybernétique de Nantes - UMR CNRS 6597

Ecole Centrale de Nantes, Nantes, France

${ }^{2}$ Laboratoire d'Acoustique de l'Université du Maine - UMR CNRS 6613

Université du Maine, Le Mans, France

Running title : Brightness of trumpet tones

Friday, March 15, 2005

\footnotetext{
${ }^{1}$ Corresponding author : Emilie.Poirson@irccyn.ec-nantes.fr
} 


\begin{abstract}
This study focuses on a particular attribute of trumpet tones; the brightness and on the physical characteristics of the instrument thought to govern its magnitude. We carried out, on the one hand, an objective study with input impedance measurements and on the other hand a subjective study with hearing tests and a panel of subjects. To create a set of different trumpets, we developed a variable depth mouthpiece whose depth can be easily and continuously adjusted from "deep" to "shallow". Using this mouthpiece and the same trumpet, we generated several instruments which may be played in three ways: (i) by a musician, (ii) by an artificial mouth, (iii) using physical modelling simulations.

The influence of the depth of the mouthpiece on the perception of the trumpet's tones was investigated, and the ability of a musician, the artificial mouth, or physical modelling simulations to demonstrate perceptively noticeable differences was assessed. Physical characteristics extracted from the impedance curves are finally proposed to explain the brightness of trumpet tones. As a result, the physical modelling simulations now seem to be mature enough to exhibit coherent and subtle perceptual differences between tones. This opens the door to virtual acoustics for instrument makers.
\end{abstract}

PACS number: $43.75 \mathrm{Fg}$ 
Poirson, JASA

\section{I - INTRODUCTION}

Studying the quality of musical instruments is particularly interesting to help their development and to improve quality assessment procedures.

In the existing literature, two kinds of studies tackle the quality assessment of musical instruments. On the one hand, there are subjective studies which aim at characterizing the subjective response given by a musician or a listener. The main difficulties with these approaches are that the subjective answers of a "subject" are generally non reproducible, semantically ambiguous, and depend on cultural and training aspects of the subject. Several studies using user-tests and sensory analysis techniques are proposed (Pratt and Bowsher, 1979). On the other hand, there are objective studies which aim at ascertaining which physical measurements seem to govern the subjective quality of the instrument (Pratt and Bowsher, 1978).

In parallel, in recent years, physical modelling simulations (Adachi and Sato, 1995) have provide interesting results to understand the functioning of brass instruments which could be used by instrument makers in the development process of new instruments. Assessing certain attributes of the quality of an instrument on a virtual prototype and using simulated tones have been proposed by Farina and Tronchin (2000).

Concerning the brass instruments, the main physical measurement is the input impedance of the bore. In order to propose a model for predicting certain qualities of brass instruments, the approach consists in discovering correlations for a set of instruments between the subjective response (given by the subject) and measurements (extracted from the impedance curve). These experiments are difficult to carry out, because one must finely control which 
parameters vary between the set of instruments, in order to be sure that the differences observed in the subjective assessment are effectively due to these variations. Plitnik and Lawson (1999) test several assumptions for the correlations between subjective attributes of horns and information extracted from the impedance curve. The conclusion is that searching for a one-to-one correspondence between a subjective attribute and an acoustic variable (coming from the input impedance) is counterproductive if not futile. These conclusions are the starting point of our study. Other studies have already been carried out to establishing the influence of the mouthpiece on the sound perceived. The influence of the mouthpiece cup volume and the impedance peaks no longer need to be proved (Benade, 1976). Wright and Campbell (1998) attempt to relate timbral changes perceived by the human ear-brain system to specific features of mouthpiece design. Psycho-acoustical listening tests have also been carried out (Carral and Campbell, 2002), to evaluate the perceived difference in timbre related to a change of mouthpiece and to find a threshold of distinguishability. They show that a small change (less than 5\%) influence the signals sufficiently to be then perceived by a subject.

Let us underline that in these studies, a lot of parameters vary from one mouthpiece to the next, and one cannot identify which parameter is the most influential on the subjective evaluation. In order to isolate and finely control the influencing variables of the timbre quality of brass instruments, we developed a trumpet mouthpiece with a depth that can be easily and continuously adjusted from "deep" to "shallow". Using this device and the same trumpet, we generated a set of instruments with notably different acoustical behaviour, varying only the internal geometry of the mouthpiece. This set of instruments has been evaluated in two ways : (1) a particular attribute of tones, (played by a musician and an 
artificial mouth, or numerically simulated), the "brightness", has been assessed via hearing tests (Petiot et al, 2003), (2) the input impedance of the instruments has been measured.

The objectives of this paper are:

- To show how a change in the depth of the mouthpiece influences the impedance curve and the perception of the brightness of tones,

- To study to what extent tones generated by a musician, an artificial mouth, or by physical modelling simulations, are similarly perceived. The question here is to know if the perception of numerically simulated tones is in accordance with the perception of tones played by a musician or an artificial mouth, and if simulations are mature enough to exhibit subtle perceptual differences between sounds,

- To find indicators extracted from the impedance curve correlated with the assessment of brightness, in order to predict this subjective assessment by objective measurements, and finally help the design of instruments.

Section 2 presents the experimental devices and the procedures used for the study. The input impedance set-up is briefly described and the procedures used to generate the trumpet tones are explained. We put particular emphasis on the method used for physical modeling simulations. The hearing tests which have been carried out are explained.

Section 3 describes the results concerning the subjective study (hearing tests) and the objective measurements (input impedance). The subjective data are analyzed by sensory analysis techniques. 
Section 4 focuses on the interpretation of the results and on the correlations between subjective evaluations and objective measurements which can be found. Conclusions and perspectives are proposed in section 5.

\section{EXPERIMENTAL DEVICES AND PROCEDURES}

\section{A. Brass instrument}

\section{The variable depth mouthpiece}

The mouthpiece of the trumpet, and more generally of all brass wind instruments, plays a paramount role both for the overall "comfort" of the musician and the sound produced. It is however difficult to investigate the correlations between the geometry and the sound produced. If one tries to explain the perceived differences, many parameters vary at the same time (the shape of the cup, thickness of the rim, width of the throat, the back bore...) (Plitnik and Lawson, 1999). For this reason, we developed a special mouthpiece for which a single geometric parameter, the depth of the mouthpiece cup, can be controlled. The system is made of a micrometer screw which increases the mouthpiece cup depth by $0.5 \mathrm{~mm}$ each turn (Figure 1). The mouthpiece can evolve easily and continuously from position T0 "shallow" (0 turn, $0 \mathrm{~mm}$ ) to position T10 "deep" (10 turns, $5 \mathrm{~mm}$ ) The mouthpiece is designed in such a way that the variation between position $\mathrm{Ti}$ and $\mathrm{T}(\mathrm{i}+1)$ is equivalent to the addition to the bore of a $0.5 \mathrm{~mm}$ thick cylinder. Eleven positions of the mouthpiece, from T0 (shallow) to T10 (deep) have been retained for the study (beyond 10 turns, the instrument becomes greatly denatured to remain playable). Using this mouthpiece, and the same trumpet (Yamaha model 1335), we generate a family of 11 different instruments, named $\mathrm{Ti}$ ( $\mathrm{i}=0$ to 10, number of turns). 
This family of instruments is the reference set for the subjective tests and the objective measurements presented in the next paragraphs.

\section{Input impedance set-up}

Brass wind instruments and more generally wind instruments can be characterised by their input impedance. Input impedances of the trumpet described above have been measured using a set-up based on one pressure and one volume velocity transducer, the source signal being a swept sine (apparatus in (Dalmont and Bruneau, 1991); for an overview of impedance measurement, (Dalmont, 2001) for example). Figure 2 presents examples of input impedance curves (magnitude and phase) of the trumpet with no valve depressed according to two cup depths, "shallow" (T0 configuration) and "deep" (T10 configuration) as described in the previous section 1.1.

Two kinds of measurements have been made. The first, called "narrow band measurement", corresponds to measurements made step by step from $50 \mathrm{~Hz}$ to $1500 \mathrm{~Hz}$, using a step equal to $1 \mathrm{~Hz}$ (Figure 2). The second, called "broad band measurement", corresponds to measurements made from $4 \mathrm{~Hz}$ step by step to $4096 \mathrm{~Hz}$ using a step equal to $4 \mathrm{~Hz}$. The narrow band measurements are used in order to extract the resonance frequencies and their associated parameters as the quality factor and the impedance magnitude value (section III.1.1). The broad band measurements are used as an input parameter of the simulated tones (section II.2.2).

A set of the input impedances corresponding to all the configurations of the mouthpiece cup is needed. Each input impedance is calculated from the measured impedance $Z_{0}(j \omega)$ of the "shallow" configuration T0. The calculation is based on the electro-acoustical line theory by 
adding to the bore a cylindrical element of the same diameter as the input diameter of the mouthpiece. The calculated impedance $\mathrm{Zc}_{\mathrm{i}}(\mathrm{j} \omega)$ is given from $\mathrm{Z}_{0}(\mathrm{j} \omega)$ by the following formula (Eq.(1)):

$$
Z c_{i}(j \omega)=\frac{Z_{o}(j \omega)+j \cdot \tan \left(k \cdot L_{i}\right)}{1+j \cdot \tan \left(k \cdot L_{i}\right) \cdot Z_{o}(j \omega)}
$$

where $L_{i}$ is the length of the added cylindrical tube, $\omega$ the angular frequency and $k$ the wave number. For the present study, $\mathrm{L}_{\mathrm{i}}$ is equal to $0.5 \mathrm{~mm}$ (case $\mathrm{i}=1$ ), $1.0 \mathrm{~mm}$ (case $\mathrm{i}=2$ ), .. 5.0 $\mathrm{mm}$ (case $\mathrm{i}=10$ ). Then a set of 10 calculated impedances is available from the measured one. Notice that the calculated impedance $\mathrm{Zc}_{10}$ has been compared to a measurement corresponding to the "deep" configuration in order to check that the calculation method is sufficiently accurate.

\section{B. Generation of tones}

\section{The artificial mouth and the trumpet player}

Several authors have worked on artificial mouths because they are essential devices to produce reproducible measurements (Gilbert et al., 1998). It is possible to finely control the embouchure and this makes it possible to take extensive measurements during stable playing conditions. It has been proved (Petiot et al., 2003) that the artificial mouth is able to exhibit differences between similar instruments via objective measurements and, in this way, may be used as a test bench for instrument makers.

For our study, several categories of tones, produced either by the artificial mouth or by a musician, were recorded. All the recordings (sampling frequency $44100 \mathrm{~Hz}, 16$ bits) were made in the same room (same place, same temperature) with a Sennheiser e604 microphone. 
The microphone was placed in the axis of the bell $($ distance $=10 \mathrm{~cm})$ and connected to the preamplifier and the Digigram Vx Pocket V2 sound card. The dynamic of the sound was measured with a sound level meter placed at the output of the bell.

For the eleven positions of the mouthpiece (T0 to $\mathrm{T} 10$ ), two notes, $\mathrm{Bb} 3$ and $\mathrm{C} 4$, were recorded. Three categories of tones were considered:

- AM : played by the Artificial Mouth, with a dynamic forte (115 dB)

- MF : played by the musician, dynamic mezzo forte $(100 \mathrm{~dB})$

- MP : played by the musician, dynamic piano $(80 \mathrm{~dB})$

A $600 \mathrm{~ms}$ window of the signal, stable in pitch and amplitude, were selected from the recordings. Each tone was normalized in duration $(600 \mathrm{~ms})$, in intensity and in frequency (frequency corresponding to the note of the well-tempered scale) with a signal processing software. A linear attack and decay of 50ms were added to the tones.

In order to limit as much as possible the variability inherent to the musician, he was asked to play the note in the easiest and most natural way (without trying to adjust the height or the timbre of the tones).

\section{Simulated tones}

Besides musical tones played by musicians or an artificial mouth, simulated tones can be obtained by finding approached solutions of acoustic models of the trumpet under playing conditions. Many authors have dealt with modelling wind instruments in order to construct a computer simulation for synthesis (called physical modelling synthesis) or to understand the physical behaviour of these instruments. The first wind instrument time-domain simulation to appear in the literature has been the clarinet model by Schumacher (1981). This method 
was adapted in order to study real instruments by using measured impedance as an input parameter of the simulation (see for example (Gazengel, 1995)). An alternative to the timedomain approach is a simulation characterising the periodic steady state oscillation using a calculation of their spectrum. This asymptotic method based on the harmonic balance technique was first used by Schumacher (1978) and by Gilbert (1989). Because the harmonic balance technique can easily show the evolution of the solutions versus a parameter of the physical model, this technique is used to study evolution of the spectrum of the simulated tones versus the cup's depth parameter of the trumpet mouthpiece.

Basically the harmonic balance technique is a numerical method computing converging periodic solutions $\mathrm{p}(\mathrm{t})$ of a system while taking a given finite number $\mathrm{N}$ of harmonics into account in a truncated Fourier serie (Eq.(2)):

$$
\begin{aligned}
p(t) & =\sum_{n=-N}^{N} C_{n} \cdot e_{2 \pi j n F_{o} t} \\
& =C_{o}+\sum_{n=1}^{N}\left\{2 \cdot \operatorname{Re}\left(C_{n}\right) \cdot \cos \left(2 \pi j n F_{o} t\right)-2 \cdot \operatorname{Im}\left(C_{n}\right) \cdot \sin \left(2 \pi j n F_{o} t\right)\right\} \\
& =C_{o}+\sum_{n=1}^{N} A_{n \cdot} \cdot \cos \left(2 \pi j n F_{o} t+\varphi_{n}\right)
\end{aligned}
$$

The unknowns of the system are the harmonic amplitudes $\left(\mathrm{C}_{0}\right.$, the real part and the imaginary part of the $N$ complex values of $C_{n}$; the alternative real unknowns $A_{n}$ and $\varphi_{n}$ can be used too) and the operating frequency $F_{o}$ (details of the technique in (Gilbert et al., 1989)).

The system of equations comes from an acoustic model of a brass instrument adapted from Elliot and Bowsher (1982) and detailed in Cullen (2000), $\mathrm{p}(\mathrm{t})$ the input mouthpiece pressure being an approached periodic solution. The acoustic model can be summarised as a system of three equations, function of three periodic variables; the opening height $\mathrm{H}(\mathrm{t})$ between the 
two lips of the player, the volume flow $v(t)$ at the entrance to the mouthpiece, and $p(t)$. The lips act as a valve controlling the air flow $\mathrm{v}(\mathrm{t})$ from the mouth (at pressure $\mathrm{P}_{\mathrm{m}}$ ) to the instrument mouthpiece (at pressure $\mathrm{p}(\mathrm{t}))$; this flow depends primarily on the pressure drop $\Delta \mathrm{p}=\mathrm{P}_{\mathrm{m}}-\mathrm{p}(\mathrm{t})$, and secondly on the opening height $\mathrm{H}(\mathrm{t})$. The behaviour of the valve can be represented by a non-linear equation (Eq.(3)) of the form :

$$
v(t)=b . H(t) \sqrt{\frac{2\left(P_{m}-p(t)\right)}{\rho}}
$$

where $b$ is an effective width of the lip's opening area. Notice that the opening height $H(t)$ varies around a rest value $\mathrm{H}_{\mathrm{o}}$ which is characteristic of the trumpet player embouchure. The simplest model which represents the lip motion is based on a one degree of freedom mechanical system represented by the following equation (Eq.(4)):

$$
\frac{d^{2}}{d t^{2}} H(t)+\frac{\omega_{L}}{Q_{L}} \frac{d^{2}}{d t^{2}} H(t)+\omega_{L}^{2} H(t)=\frac{P_{m}-p(t)}{\mu_{L}}
$$

where $\omega_{\mathrm{L}}$ and $\mathrm{Q}_{\mathrm{L}}$ are the resonance (angular) frequency and the quality factor of a mechanical resonance of the lips. The parameter $\mu_{\mathrm{L}}$ is an effective mass per area of the lips. The two previous equations (Eq. 3 and 4) represent the non-linear aeroelastic valve which is coupled with the air column. An effective way to model the acoustic behaviour of the air column is to write an impedance relationship in the frequency domain (Eq.(5)):

$$
P(j \omega)=Z(j \omega) \cdot V(j \omega)
$$

where $Z(j \omega)$ is the input impedance of the brass instrument, $P(j \omega)$ and $V(j \omega)$ being the mouthpiece pressure and the volume flow written in the frequency domain. 
The elementary model based on the three Eq.(3), (4) and (5) is adapted to find numerical solution of steady self-sustained oscillations of the trumpets using the harmonic balance technique. In order to do the simulations, values of the parameters used in the model are needed. If the trumpet is well described by its input impedance which is known (see section II-1.2), providing lip parameters needed in Eq.(3) and (4) is not an easy task. The lip parameters values used in the harmonic balance technique to simulate the trumpet player embouchure are listed in Table I. The values have been adapted from the study of Cullen et al (2000) and particularly from their Table III in order to get realistic and sensible simulations.

This leads to a very fine spectral analysis and is very convenient for showing the modifications of the playing frequency and the spectrum produced by the variation of the trumpet mouthpiece cup geometry (e.g., a change of the input impedance associated to each mouthpiece geometry), the lip parameters being constant during all the simulations. Eleven simulated acoustic pressure associated to the eleven input impedances T0 to T10 are calculated.

The approached solutions $\mathrm{p}(\mathrm{t})$ coming from the elementary model are corresponding to the instrument mouthpiece acoustic pressure. Now external acoustic pressures are needed in order to be comparable with the recorded trumpet tones described in the previous section. Then the concept of "spectrum transformation function" was used. According to Benade's theory (1976), the general trend of the spectrum transform function $\mathrm{T}(\mathrm{j} \omega)$ relating the external pressure amplitudes of the various harmonics to the internal pressure amplitudes being a linear relationship varying from 0 to 1 as a function of frequency from $0 \mathrm{~Hz}$ to a cut- 
off frequency $f_{c}$, we have chosen $f_{c}$ equal to $1400 \mathrm{~Hz}$. Then above $f_{c}, T(j \omega)$ is assumed to be equal to 1 , the spectrum transform function representing an ideally high-pass filter. At the end, the solutions of the harmonic balance simulation are given by a set of external pressure amplitudes of the harmonics. To be used during the perceptive tests, the eleven solutions defined in the frequency domain have to be recalculated in the time domain by adding all the harmonics - Fourier theorem -, and are windowed and normalised by using the method applied to the recorded tones (see section II-2.1).

The method was then used to generate simulated tones (Simu) corresponding to our set of instruments ( $\mathrm{T} 0$ to $\mathrm{T} 10)$, by using as input parameter of the simulation the measured impedance.

\section{Subjective tests}

The subjective tests consisted in psychoacoustical hearing tests and have involved several subjects and stimuli. A detailed presentation of the various hearing tests we have carried out with the variable depth mouthpiece is presented by Petiot et al (2004). We will describe in this section how the stimuli were designed, and the tests we have focused on.

\section{Stimuli of the tests}

The stimuli of the tests are made of tones belonging to the following categories:

- AM : played by the Artificial Mouth, with a dynamic forte (115 dB)

- MF : played by the musician, dynamic mezzo forte $(100 \mathrm{~dB})$

- MP : played by the musician, dynamic piano $(80 \mathrm{~dB})$

- Simu : numerically simulated tones 
Poirson, JASA

The subjects listened to the stimuli via a headphone Audio-Technica ATH-M40

\section{Test 1: threshold of distinguishability}

The variations of the bore of the instruments (T0 to T10) being very weak, the aim of this first test was to estimate if differences of timbre corresponding to different instruments can be perceived. The assessment of the difference was done by pairwise comparison. The objectives of this test were firstly to evaluate, for each category of tones, the threshold of distinguishability of the sounds differentiation, and secondly to show if the value of this threshold depends on the category.

In order to limit the duration of the tests, only 5 sounds (corresponding to positions T0, T2, T5, T6, T10) were selected, for each category. All the 10 possible pairs among the 5 sounds were considered. A typical stimulus of Test 1 (e.g. Bb3, AM T0-T6) consists of a pair of sounds of the same category (e.g. AM, MF, MP or Simu) and corresponding to the same note, separated by $600 \mathrm{~ms}$ of silence. In order to check the coherency of the subject, one pair of identical sounds (T5-T5) was added to the stimuli. A total of 11 pairs of sounds, for each category (AM, MF, MP or Simu), constituted the stimuli of the tests. The pairs proposed for the evaluation are marked with a cross in the following matrix (Table II).

The pairs were presented three times to the subject in a random order, so as to check the consistency of the subjects.

The subjects were asked to indicate whether or not the two tones of a pair were different. They could listen to the pair as many times as they wished, and gave their answers by clicking on boxes via an interface programmed in VisualBasic6 (Figure 3).

Due to time constraints, the test 1 was performed in two parts. 
In a first session, subjects assessed all the pairs of the AM, MF and MP categories, for the note $\mathrm{Bb}^{2} .99$ pairs (11 pairs $\times 3$ categories $\times 3$ replications $)$ was proposed in this session.

In a second session, subjects assessed all the pairs of the Simu category, for the same note Bb3 (total of 33 pairs in this session: 11pairs $\times 1$ categories $\times 3$ replications). A total of 20 subjects (including 12 musicians), performed the test 1 . In addition to these evaluation, subjects were asked to indicate at the end of the assessments the more relevant terms to describe the differences between tones.

\section{Test 2: ranking on a gradual axe of brightness}

After a study of the differences between tones (test 1), we examined the nature of these differences (test 2).

We focused for test 2 on a particular attribute of the timbre: the brightness of the sound (Krimphoff, 1993; Grey, 1977). Psychoacoustic studies have shown that this dimension is one of the main perceptual dimensions of the timbre in permanent regime. The tones having been previously balanced in pitch and intensity, the differences are based on the timbre only. Furthermore, to the question asked at the end of test $\mathbf{1}$ "which word qualifies for you these differences", brightness was one of the most recurrent term.

For the categories of tones MF, AM and Simu, with the eleven tones T0 to T10 as input, the subjects were asked to rank them on a graduate scale from "dull" to "bright", with a "click and drag" interface programmed in VisualBasic6.

\footnotetext{
${ }^{2}$ The test has been done with the note $\mathrm{C} 4$ too. The results were quite similar, and are not presented here.
} 
The objective of test $\mathbf{2}$ is firstly to know if differences of brightness can be significantly detected, to check the existence of a correlation between the subjective assessment of brightness and the depth of the mouthpiece, and finally to see if the correlation depends on the category of tones.

The experimental data and results are presented in the following section.

\section{EXPERIMENTAL DATA AND RESULTS}

\section{A. Subjective study}

\section{Test 1 : threshold of distinguishability}

First, three subjects, suspected to be non coherent because they assessed at least once the pair of identical tones as "different", were excluded from the final results.

Next, for the remaining 17 subjects and for each pair of tones, we assigned a " 1 " for a correct answer and " 0 " for incorrect. Subjects' answers are cumulated and expressed in percentages: thus, $100 \%$ means that all the subjects found that, each time they heard it, the pair of tones was "different", $0 \%$ means that the pair has always been evaluated as similar by all the subjects. The results for each category of sounds are shown in Table III.

The results show the same tendency for all categories of tones: the larger the difference in the mouthpiece depth, the higher the percentage of correct answers, i.e more the tones are perceived as different. The greater the difference of mouthpiece depth, the better the differentiation of timbre. The results indicate in particular that the importance of the perceived differences between the simulated tones vary in the same way for the tones played by a musician as by the artificial mouth. Simulation is able to reproduce differences between 
sounds which are similar to those played by the musician or the artificial mouth. This result, not obvious at all in the beginning of the study, is a first important conclusion.

In order to evaluate the threshold of distinguishability for each category of tones, two criteria are next calculated.

The first one is based on a statistical test. Given that our test is based on a forced choice question (2 possible answers, "identical" or "different"), we have to check the risk of obtaining such results by chance. The statistical law adapted in this case is the binomial law ${ }^{3}$, which gives in our case that up to $55 \%$ of correct answers, the risk of obtaining this score at random is less than 5\%. The pairs for which the risk is greater than $5 \%$ are shaded in the Table III, the number of pairs concerned is indicated in Table IV, column 2.

The second criteria is the average percentage of correct answers for all the tested pairs.

These two criteria are given for each category of tones in Table IV.

Given that the higher the average percentage of correct answers and the lower the number of removed pairs, the higher the threshold of distinguishability, we can conclude that the ranking of the categories according to this threshold is:

\section{thres $_{\mathrm{AM}}<$ thres $_{\mathrm{MF}}<$ thres $_{\mathrm{Simu}}<$ thres $_{\mathrm{MP}}$}

The threshold of differentiation is the lowest with the AM. Thus, the artificial mouth seems to be a good device to exhibit subtle differences between instruments.

Perceived differences for MP (musician, dynamic $p$ ) are less marked than for the other categories. The musician seems to "smooth" the differences between the instruments, and

\footnotetext{
${ }^{3}$ the probability to have $k$ successes in a fixed number $n$ of independent random trials, all of which have the same probability $p$ (here $p=0.5$ ), is given by ${ }_{n} C_{k} p^{k}(1-p)^{n-k}$, with ${ }_{n} C_{k}=n ! /(k !(n-k) !)$.
} 
furthermore the dynamic "piano" is not favourable to reveal differences of timbre (the dynamic piano does not allow the generation of high order harmonics).

This first test shows finally that subjects perceived differences between sounds even for small variations of the bore of the instrument. For the case of MP, differences were so small (the threshold is high) that we decided to exclude this category for the test 2.

\section{Test 2 : Ranking on an axe of brightness}

Each subject provided a ranking of the tones on an axe of brightness. Before aggregating the assessments of the subjects in an average answer, we have first to check whether or not the group of subjects is homogeneous, and to select a panel of subjects who agree enough in their evaluation. The inter-subject Spearman coefficient $r_{a b}$ between two subjects is a measurement of the agreement between their rankings:

$$
r_{a b}=1-\frac{6 \cdot \sum_{i=1}^{n}\left(C_{a}\left(T_{i}\right)-C_{b}\left(T_{i}\right)\right)^{2}}{n \cdot\left(n^{2}-1\right)}
$$

with n: number of tones (here 11)

$\mathrm{C}_{\mathrm{a}}$ and $\mathrm{C}_{\mathrm{b}}$ : rankings proposed by subject $a$ and subject $b$

This coefficient is equal to 1 if the rankings are exactly the same, -1 if they are exactly opposite. A cluster analysis (hierarchical ascendant classification) on the distance matrix between all the subjects (distance $=1-\mathrm{r}_{\mathrm{ab}}$ ) has been done for each category of tones. It reveals that the group of subjects was not necessarily homogeneous. 
For the category AM, three subjects were discordant (atypical), and proposed roughly reverse rankings relative to the rest of the group ${ }^{4}$. The rest of the group agreed in the assessment and constituted an homogeneous panel.

For the category Simu, all the subjects agreed in their assessments.

For the category MF, the answers were very disparate: after an analysis of the dendrogram, we selected a panel of 7 subjects but the agreement between them is rather poor, so the average value will be suspected as unreliable.

In order to exhibit a specific tendency of the panel of subjects in the assessment of brightness, we removed the data of the atypical subjects and we considered only the data of the panel to get an average answer. Table V presents for each instrument and each category of tones the average rank of brightness (brightness score).

For AM and Simu, the brightness score is highly correlated with the mouthpiece depth. For MF, this correlation is less marked. This could be explained by the fact that the musician compensates the difference of mouthpiece and adapts his embouchure to the instrument in order to produce the sound he has in mind.

The Friedman statistic test on the average ranks shows that, for all categories of sounds (AM, MF and Simu), there is globally a significant difference between the sounds according to their brightness with a p-value $<1 \%$.

Next, for each pair of sounds, the Friedman test shows that some pairs are significantly perceived as different with a p-value $<5 \%$. These pairs are shaded in Table VI. For the other pairs (e.g. T0-T2), the difference of brightness is too small to be statistically significant.

\footnotetext{
${ }^{4}$ we cannot say if these 3 subjects had actually understood the instruction, had a defective hearing or have really judged that in a different way.
} 
For the AM and Simu categories, the results show that, the greater the difference of mouthpiece depth, the more the difference of brightness is significantly perceived. It has to be noticed that the perceived brightness of the simulated tones is coherent with the perceived brightness of real tones (AM). This is a strong point for the validation of simulations.

For the MF category, the previous tendency for the perceived difference of brightness is less obvious. The variability created by the musician is of the same order as the subtleties that we want to explain. For this reason, in order to interpret the difference of brightness with objective variables, we will focus for the rest of our work on sounds produced by the artificial mouth.

\section{B. Objective study}

\section{From the instrument}

\section{The resonance frequencies}

Given that we focused our subjective study on a particular tone of the trumpet (partial\#4), additional variables of the impedance curve related to the $4^{\text {th }}$ and $8^{\text {th }}$ resonance peaks have been extracted.

In the playing situation, the musician produces a note whose frequency - the playing frequency - is close to the resonance frequency of the vibration mode that controls the selfsustained excitation process. The resonance frequencies can be defined as the frequencies corresponding to the maximum - peak amplitude - of the input impedance magnitude of the air column of the instrument with its input section closed. Then the resonance parameters are directly extracted from the "narrow band measurement" (see section II-1.2), the frequency accuracy being equal to the frequency sampling, it means $1 \mathrm{~Hz}$. Another way of defining the 
resonance and to extract the resonance parameters (resonance frequency, quality factor and magnitude) from the measured impedance $Z(j \omega)$ is to fit locally around the resonance number $\mathrm{n}$ the experimental result with a model of resonance like the Lorentz model as follows (Eq.(7)):

$$
Z(j \omega)=G 0_{n}+G 1_{n} \frac{j \cdot\left(\omega / 2 \pi \cdot \text { fres }_{n}\right) \cdot Q_{n}^{-1}}{-\left(\omega / 2 \pi \cdot \text { fres }_{n}\right)^{2}+j \cdot\left(\omega / 2 \pi \cdot \text { fres }_{n}\right) \cdot Q_{n}^{-1}+1}
$$

where fres $\mathrm{n}_{\mathrm{n}}$ and $\mathrm{Q}_{\mathrm{n}}$ are the resonance frequency and the quality factor of the $\mathrm{n}^{\text {th }}$ resonance, the complex number $\mathrm{G} 0_{\mathrm{n}}$ and the real positive value $\mathrm{G} 1_{\mathrm{n}}$ being useful to get the magnitude of the input impedance at the frequency fres $\mathrm{n}_{\mathrm{n}}$. More precisely, the individual peak number $\mathrm{n}$ is selected and the complex input impedance is locally fitted with a circle in the Nyquist complex plane using a least-squares minimisation - for a review of the curve-fitting methods, see for example (Ewins, 1984). Even if the frequency accuracy is not easy to determine, by circle-fitting based on four experimental data we assume that it is less than $0.1 \mathrm{~Hz}$.

The resonance frequencies were extracted from the impedance curves.

Because the subjective study (see section III) is focused on a particular tone of the trumpet the partial number 4 - the frequencies, the Qvalues and the magnitudes associated to the $4^{\text {th }}$ and $8^{\text {th }}$ resonance peaks are especially needed and listed in Table VII - notice that the inharmonicity $f_{\text {res } 8} / 2 f_{\text {res } 4}$ is given too. The resonance parameters of the eleven following input impedances $-\mathrm{T} 0, \mathrm{~T} 1, \mathrm{~T} 2, \ldots$ and $\mathrm{T} 10$ - are extracted.

\section{From the tones}

\section{Spectral Centroid :}


The spectral centroid is commonly associated with the measure of the brightness of a sound (Grey and Gordon 1978). This measurement is obtained by evaluating the "centre of gravity" using the Fourier transform's frequency and magnitude information Table I, where $A_{n}$ represents the amplitude of harmonic $\mathrm{n}$ of the spectrum and $\mathrm{N}$ the number of harmonics considered (in our case, $\mathrm{N}=6$ ).

$$
S c=\frac{\sum_{n=1}^{N} n \cdot A n}{\sum_{n=1}^{N} A n}
$$

Table VIII shows for each position of the mouthpiece the $S c$ of the tones, for 3 categories of tones (AM, MF, Simu).

For the categories AM and Simu, we notice that the deeper the mouthpiece, the lower the Sc. This behaviour is not true for the category MF. This can be explained by the fact that the musician is not reproducible enough (his embouchure varies according to the instrument played). The musician produces variations of the timbre of the same order as those which are created while varying the mouthpiece, and this phenomenon certainly disrupts and masks the effect of the mouthpiece on the spectral content of the tones.

\section{Interpretation of the results}

\section{Correlation between the brightness and the spectral centroid}

The correlation between the brightness scores of the tones (Table V) and the spectral centroid (Table VIII) has been studied. For each category of tones, the linear correlation coefficient (Pearson) is given Table IX. 
For $\mathbf{A M}$ and Simu, the results are in agreement with a well-known result in psychoacoustics: the brightness of the sounds is correlated with their spectral centroid (Krimphoff et al, 1994). This result gives credit to our procedure of brightness assessment. For MF, there is surprisingly no correlation $(\mathrm{R}=-0.04)$. In fact, the agreement between the subjects of the panel in their brightness assessment is probably too weak, so the average scores of brightness are not reliable and representative of a tendency.

\section{Correlation between the brightness and the resonance parameters of the instrument}

The study of sound production in brass instruments shows that there is a coupling between the lips of the musician and the resonator. The intonation of the instrument is not only controlled by the closest resonance frequency of the input impedance but possibly conditioned by upper resonance frequencies of the resonator.

In order to study the brightness of the note $\mathrm{Bb} 4$, we make the assumption that the spectrum and then the spectral centroid of the note in permanent regime is mainly governed by the characteristics of the impedance curve corresponding to the partial $n^{\circ} 4$ and $n^{\circ} 8$ of the impedance curve (the resonance frequency $n^{\circ} 8$ corresponds roughly to the first harmonic of the note $\mathrm{Bb} 4)$. Our objective is to find which characteristics of the impedance curve are the more influencing on the Spectral centroid $S c$ of the tones, and then on their brightness (the relation between the brightness and the amplitude of the high frequency components of the spectrum is clearly demonstrated).

We considered the following variables of the impedance curve: $f_{\text {res } 8} / 2 f_{\text {res } 4},\left|Z_{8}\right| /\left|Z_{4}\right|, Q_{8} / Q_{4}$. Figure 4 shows a superimposed representation of the characteristics of the impedance curve around the resonances $\mathrm{n}^{\circ} 4$ and $\mathrm{n}^{\circ} 8$, and of the fundamental $f$ and first harmonic $2 f$ of the note 
$\mathrm{Bb} 4$. As a first approximation we suppose that the playing frequency of the note $(f)$ is equal to the resonance frequency $f_{\text {res } 4}$, and we suppose that the inharmonicity $f_{\text {res } 8} / 2 f_{\text {res } 4}$ is less than 1 , in other words the resonance $\mathrm{f}_{\text {res } 8}$ is slightly smaller than the first harmonic $2 \mathrm{f}$ (Figure 4 ). A physical analysis based on the input impedance curve schematically displayed Figure 5 indicates the following sensible conclusions:

- The amplitude of the first harmonic of Bb4 (frequency $=2 \mathrm{f}$ ) is favoured if the inharmonicity $\mathrm{f}_{\text {res } 8} / 2 \mathrm{f}_{\text {res } 4}$ increases to 1 , all other parameters of the impedance curve being constant. So, the $S c$ of a tone is favoured if $\mathrm{f}_{\text {res } 8} / 2 \mathrm{f}_{\text {res } 4}$ increases until 1 ,

- The amplitude of the first harmonic of $\mathrm{Bb} 4$ is favoured if the magnitude of $\mathrm{Z}_{8}$ increases, all other parameters of the impedance curve being constant. So, the $S c$ of a tone is favoured if $\left|Z_{8}\right| /\left|Z_{4}\right|$ increases,

- The amplitude of the first harmonic of $\mathrm{Bb} 4$ is favoured if the quality factor $\mathrm{Q}_{8}$ decreases, all other parameters of the impedance curve being constant. So, the $S c$ of a tone is favoured if $\mathrm{Q}_{8} / \mathrm{Q}_{4}$ decreases,

Of course, things are not so simple when we want to interpret the values of the brightness of the tones using characteristics of the impedance of our set of instruments: all the characteristics vary at the same time and their influence is combined. For example, the linear correlation coefficient (Pearson, given Table X) between the brightness scores of the tones (Table V) and $\left|Z_{8}\right| /\left|Z_{4}\right|$ on the one hand and $\left|Q_{8}\right| /\left|Q_{4}\right|$ on the other hand confirm the suggested conclusions above. On the other hand, the correlation between the inharmonicity $\mathrm{f}_{\text {res } 8} / 2 \mathrm{f}_{\text {res } 4}$ is negative and seems to be counterintuitive with the physics of the instrument: greater the inharmonicity, brighter the sound. 
In fact, this negative correlation is quite coherent because many underlying characteristics of the impedance varied and they are not taken into account with a simple linear correlation. In order to reveal the underlying variables among the 3 variables $\left(f_{\text {res } 8} / 2 f_{\text {res } 4},\left|Z_{8}\right| /\left|Z_{4}\right|\right.$, $\left.\left|\mathrm{Q}_{8}\right| /\left|\mathrm{Q}_{4}\right|\right)$ we performed a principal component analysis on the standardised data (correlation matrix) concerning 11 individuals and the 3 variables (from Table VII). The first factor accounts for $92 \%$ of variance, the second factor for $7 \%$. So a representation on a plane accounts for $99 \%$ of variance. Figure 5 represents the position of the individuals (T0 to T10) in the factorial plane, and the directions of the initial variables $\left(f_{\text {res } 8} / 2 f_{\text {res } 4},\left|Z_{8}\right| /\left|Z_{4}\right|\right.$, $\left.\left|\mathrm{Q}_{8}\right| /\left|\mathrm{Q}_{4}\right|\right)$. The evolution of the variables is actually complex and when $\mathrm{f}_{\text {res } 8} / 2 \mathrm{f}_{\text {res } 4}$ increases, $\left.\left|\mathrm{Q}_{8}\right| /\left|\mathrm{Q}_{4}\right|\right)$ increases and $\left|\mathrm{Z}_{8}\right| /\left|\mathrm{Z}_{4}\right|$ decreases.

In order to interpret the brightness scores, we performed a multiple linear regression of the

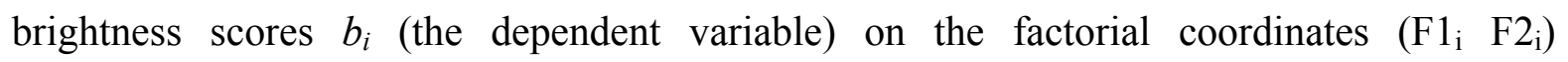
(independent variables). This classical technique in sensory analysis leads to the determination of the coefficients $(\alpha, \beta)$ of the regression, given by:

$$
b_{i}=\alpha \cdot F 1_{i}+\beta \cdot F 2_{i}+\gamma
$$

The determination coefficient $\mathrm{R}^{2}$ of the regressions are given in Table XI.

A very good adjustment is observed for $\mathbf{A M}$ and Simu (significant with p-value $<1 \%$ ). For MF, only $41 \%$ of the variance is taken into account by the regression. This is because the average score of brightness is in the case of MF certainly not reliable.

For the $\mathbf{A M}$ and Simu categories, a graphic interpretation of the regression can be given by plotting in the factorial plane the vector model of the attribute "brightness", B.AM and B.Simu. (Figure 5). The origin of the vector is arbitrarily located in the origin of the frame, 
the values of the regression coefficients $(\alpha, \beta)$ give the position of the arrow, the arrowhead points in the direction of increasing brightness. This vector is parallel to the steepest slope line of the plane (Eq.(9)) and the perpendiculars to the vector are the "iso-brightness lines". The results of the regression are entirely in agreement (vectors B.AM and B.Simu are almost merged): the brightness of simulated tones is similarly interpreted than the brightness of sounds played by the artificial mouth. This is again a strong point for the validation of simulations.

Concerning the relations between the brightness and characteristics of the impedance curve, we noticed that the higher $\left|\mathrm{Z}_{8}\right| /\left|\mathrm{Z}_{4}\right|$, and the lower $\mathrm{Q}_{8} / \mathrm{Q}_{4}$, the brighter the sound. This result is conform to the physicist's intuition presented above. But we noticed too that the lower $f_{\text {res } 8} / 2 f_{\text {res } 4}$, the brighter the sound. This result could appear to be counterintuitive, but we must keep in mind that the influence of this variable can be masked by the influence of the others. Finally, our study indicates that the more influencing variable on the brightness is $\left|Z_{8}\right| /\left|Z_{4}\right|$.

\section{CONCLUSIONS}

In this paper, we studied the brightness of trumpet tones, the tones being generated in three ways : by a musician - by an artificial mouth - by physical modelling simulations. Two studies were conducted on a set of instruments using a variable-depth mouthpiece: a subjective study with hearing tests for the assessment of the threshold of distinguishability between pairs of sounds and for the evaluation of the brightness ; an objective study with the extraction of objective variables from the impedance curve. 
We showed that the artificial mouth and the simulations give coherent results with those of the musician concerning the perception of differences of timbre. We verified that the threshold of distinguishability between sounds is of the same order for the artificial mouth, simulated tones and for the musician. We demonstrated that the artificial mouth and the simulations give similar results concerning the brightness of trumpet tones. We emphasized the fact that the musician is not reproducible enough and creates difference of timbre of the same order as the difference produced by the depth-change in the mouthpiece. We conclude that the artificial mouth is an essential device for the study of the quality of wind instrument, for the conception of new instruments, and for the validation of realistic simulated tools. Concerning the interpretation of brightness of sound, we have proposed a method based on principal component analysis and multiple regression for the research of the underlying and more influencing characteristics of the impedance curve on the brightness of sounds. We found that the magnitude of the impedance peak corresponding to the second harmonic of a tone is highly correlated with its brightness, and seems to be the cause. But we must keep in mind that correlation is not equivalent to causality. The simulation will be a useful tool to study experimentally the influence of variables of the impedance curve on attributes of the sounds. By making it possible to test a wide range of different virtual instruments, statistical studies will be possible to test assumptions concerning the causal links between objective characteristics of instruments and sound attributes. These causal links will be finally used for the design of new instruments.

Concerning the simulations, we have proved that sounds simulated by the harmonic balance technique are able to reproduce differences of timbre which are similarly perceived to those 
played by a musician or the artificial mouth. This is a first step for the use of virtual acoustics techniques in the conception of new instruments.

\section{ACKNOWLEDGMENTS}

Authors are indebted to J.L. Le Carrou for his participation in experiments and to V. Fitzpatrick for her editorial support. 
Poirson, JASA

\section{REFERENCES}

Adachi S., Sato M.A. (1995). "Time-domain simulation of sound production in the brass instrument.” J. Acoust. Soc. Am. 97 (6), 3850-3861.

Benade A. H.(1976). Fundamentals of musical acoustics. Oxford University Press, New York.

Caral S., Campbell M.(2002). "The influence of the mouthpiece throat diameter on the perception of timbre of brass instrument" + ref...ISMA Mexico.

Cullen, J.S., Gilbert, J., and Campbell, D.M. (2000). "Brass instruments : linear stability analysis and experiments with an artificial mouth.” ACUSTICA - acta acustica 48, 73-84.

Dalmont, J.P., Bruneau, A.M. (1991). "Acoustic impedance measurement : plane-wave mode and first helical-mode contributions.” J. Acoust. Soc. Am. 91, 3026-3033.

Dalmont, J.P. (2001). “Acoustic impedance measurement, part 1 : a review.” Journal of Sound and Vibration 243, 427-439.

Elliot, S.J., and Bowsher, J.M. (1982). "Regeneration in brass wind instruments", Journal of Sound and Vibration 83, 181-217.

Farina A., Tronchin L. (2000) "On the "virtual" reconstruction of sound quality of trumpets". Acustica - Acta Acustica. Vol. 86, 747-745.

Gazengel, B., Gilbert, J., and Amir, N. (1995). "Time domain simulation of a single reed wind instrument. From the measured input impedance to the synthesis signal. Where are the tramps ?" Acustica 3, 445-472.

Gilbert, J., Kergomard, J., Ngoya, E. (1989). "Calculation of the steady state oscillations of a clarinet using the harmonic balance technique.” J. Acoust. Soc. Am. 86, 35-41. 
Gilbert J., Ponthus S., Petiot J.F. (1998). Artificial buzzing lips and brass instruments : experimental results. J. Acoust. Soc. Am. 104, 1627-1632.

Grey, J.M. (1977). Multidimensional perceptual scaling of musical timbre. J. Acoust. Soc. Am. 61 (5) 1270-1277.

Krimphoff J.(1993). Analyse acoustique et perception du timbre“. DEA d'acoustique appliquée, IRCAM.

Krimphoff J., Mc Adams S., Winsberg S.(1994).Caractérisation du timbre des sons complexes. Analyses acoustiques et quantification psychophysique. Journal de physique IV, colloque C5, supplément au journal de Physique III, volume 4.

Petiot J.F., Tessier F., Gilbert J., Campbell M.(2003). Comparative Analysis of Brass Wind Instruments With an Artificial Mouth : First Results Acta Acustica. n6, Vol 89, pp 974-979.

Petiot J-F., Poirson E., Gilbert J.(2004). "Study of the brightness of trumpets' sounds by analysing the impedance curves." Proceedings of the CFA/DAGA04, 22-25 March 2004, Strasbourg, France.

Plitnik G. R, Lawson B.A. (1999). An investigation of correlations between geometry, acoustic variables, and psychoacoustic parameters for French horn mouthpieces. J. Acoust. Soc. Am. 106, 1111-1125.

Pratt R.L., Bowsher J.M. (1978). “The subjective assessment of trombone quality”. Journal of Sound and Vibration 57, 425-435.

Pratt R.L., Bowsher J.M. (1978). “The objective assessment of trombone quality”. Journal of Sound and Vibration 57, 425-435. 
Schumacher, R.T. (1978). "Self sustained oscillations of the clarinet: an integral equation approach.” Acustica 40, 298-309.

Schumacher, R.T. (1981). "Ab initio calculations of the oscillations of a clarinet." Acustica 48, 73-84.

Wright H.A.K, Campbell D.M.(1998). “ The influence of the mouthpiece on the timbre of cup-mouthpiece wind instruments.” ISMA 98, Leavenworth, Washington, USA 
Poirson, JASA

\section{TABLES}

Table I : Lip parameters used in the harmonic balance technique to simulate the trumpet player embouchure.

\begin{tabular}{|c|c|}
\hline $\mathrm{b} / \mathrm{mm}$ & 10 \\
\hline $\mathrm{H}_{\mathrm{o}} / \mathrm{mm}$ & 0.05 \\
\hline$\omega_{\mathrm{L}} / 2 \pi / \mathrm{Hz}$ & 410 \\
\hline $\mathrm{Q}_{\mathrm{L}}$ & 5 \\
\hline $1 / \mu_{\mathrm{L}} / \mathrm{m}^{2} \mathrm{~kg}^{-1}$ & -0.5 \\
\hline
\end{tabular}


Poirson, JASA

Table II : The eleven pairs proposed for the subjective evaluations

\begin{tabular}{|c|c|c|c|c|c|c|}
\hline & \multicolumn{5}{|c|}{$\begin{array}{l}\text { Instrument } \\
\text { for sound } 2\end{array}$} \\
\hline & & T0 & $\mathrm{T} 2$ & T5 & T6 & T10 \\
\hline \multirow{5}{*}{ 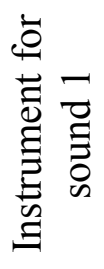 } & T0 & & $X$ & $X$ & $X$ & $X$ \\
\hline & $\mathrm{T} 2$ & & & $X$ & & \\
\hline & T5 & & & $X$ & & \\
\hline & T6 & & $X$ & $X$ & & $X$ \\
\hline & $\mathrm{T} 10$ & & $X$ & $X$ & & \\
\hline
\end{tabular}


Table III : Percentage of correct answers in the paired comparisons for test 1

\begin{tabular}{|c|c|c|c|c|c|c|}
\hline \multirow{2}{*}{\multicolumn{2}{|c|}{$\mathbf{A M}$}} & \multicolumn{5}{|c|}{$\begin{array}{l}\text { Instrument } \\
\text { for sound } 2\end{array}$} \\
\hline & & \multirow{2}{*}{ T0 } & \multirow{2}{*}{ 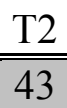 } & \multirow{2}{*}{$\begin{array}{l}\text { T5 } \\
88\end{array}$} & \multirow{2}{*}{ T6 } & \multirow{2}{*}{\begin{tabular}{|l}
$\mathrm{T} 10$ \\
100
\end{tabular}} \\
\hline 。ั & T0 & & & & & \\
\hline$\Rightarrow-$ & T2 & & & 61 & & \\
\hline$\Xi \Xi$ & T5 & & & & & \\
\hline E & T6 & & 80 & 53 & & 96 \\
\hline$\Xi$ & T10 & & 100 & 98 & & \\
\hline
\end{tabular}

\begin{tabular}{|c|c|c|c|c|c|c|}
\hline \multirow{2}{*}{\multicolumn{2}{|c|}{ MF }} & \multicolumn{5}{|c|}{$\begin{array}{l}\text { Instrument } \\
\text { for sound } 2\end{array}$} \\
\hline & & \multirow[t]{2}{*}{ T0 } & \multirow{2}{*}{ 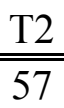 } & \multirow{2}{*}{\begin{tabular}{|l} 
T5 \\
86
\end{tabular}} & \multirow{2}{*}{$\begin{array}{l}\text { T6 } \\
98\end{array}$} & \multirow{2}{*}{$\frac{\mathrm{T} 10}{994}$} \\
\hline ö & T0 & & & & & \\
\hline$\rightleftarrows-$ & T2 & & & 76 & & \\
\hline$\Xi \Xi$ & T5 & & & & & \\
\hline$E$ in & T6 & & 69 & 41 & & 55 \\
\hline & $\mathrm{T} 10$ & & 96 & 59 & & \\
\hline
\end{tabular}

\begin{tabular}{|c|c|c|c|c|c|c|}
\hline \multirow{2}{*}{\multicolumn{2}{|c|}{ MP }} & \multicolumn{5}{|c|}{$\begin{array}{l}\text { Instrument } \\
\text { for sound } 2\end{array}$} \\
\hline & & \multirow[t]{2}{*}{$\mathrm{T} 0$} & \multirow{2}{*}{\begin{tabular}{|l|}
$\mathrm{T} 2$ \\
53
\end{tabular}} & \multirow{2}{*}{$\frac{\mathrm{T} 5}{76}$} & \multirow{2}{*}{\begin{tabular}{l|} 
T6 \\
61 \\
\end{tabular}} & $\mathrm{~T} 10$ \\
\hline$\check{0}$ & T0 & & & & & 80 \\
\hline 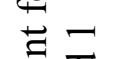 & $\mathrm{T} 2$ & & & 6 & & \\
\hline $\bar{\Xi} \Xi$ & T5 & & & & & \\
\hline$E_{\infty} \bar{D}$ & T6 & & 14 & 4 & & 59 \\
\hline & $\mathrm{T} 10$ & & 84 & 41 & & \\
\hline
\end{tabular}

\begin{tabular}{|c|c|c|c|c|c|c|}
\hline \multirow{2}{*}{\multicolumn{2}{|c|}{ Simu }} & \multicolumn{5}{|c|}{$\begin{array}{l}\text { Instrument } \\
\text { for sound } 2\end{array}$} \\
\hline & & \multirow[t]{2}{*}{ T0 } & \multirow{2}{*}{ 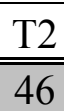 } & \multirow{2}{*}{$\frac{\mathrm{T} 5}{\mathrm{~T} 69}$} & \multirow{2}{*}{ T6 } & \multirow{2}{*}{$\begin{array}{r}\mathrm{T} 10 \\
94\end{array}$} \\
\hline & T0 & & & & & \\
\hline$\stackrel{ \pm}{\rightleftarrows}=$ & $\mathrm{T} 2$ & & & 50 & & \\
\hline $\bar{\Xi} \bar{\Xi}$ & T5 & & & & & \\
\hline$E_{\infty}^{E} \bar{\phi}$ & T6 & & 48 & 15 & & 56 \\
\hline & $\mathrm{T} 10$ & & 91 & 48 & & \\
\hline
\end{tabular}


Table IV : Criteria proposed to rank the category of sounds according to the threshold of distinguishability

\begin{tabular}{||c|c|c||}
\hline Category & $\begin{array}{c}\text { Number of pairs } \\
\text { removed by the } \\
\text { statistical test }\end{array}$ & $\begin{array}{c}\text { Average percentage } \\
\text { of correct answers }\end{array}$ \\
\hline AM & 2 & $81.5 \%$ \\
\hline MF & 2 & $73.1 \%$ \\
\hline Simu & 5 & $59.7 \%$ \\
\hline MP & 5 & $47.8 \%$ \\
\hline
\end{tabular}


Table V : Brightness score resulting of test 2

\begin{tabular}{||c||c|c|c|c|c|c|c|c|c|c|c||}
\hline Position & T0 & T1 & T2 & T3 & T4 & T5 & T6 & T7 & T8 & T9 & T10 \\
\hline \hline $\begin{array}{c}\text { Brightness } \\
\text { for AM }\end{array}$ & 10.93 & 10.07 & 9.00 & 6.60 & 5.87 & 7.67 & 4.47 & 3.07 & 3.20 & 3.33 & 1.60 \\
\hline \hline $\begin{array}{c}\text { Brightness } \\
\text { for Simu }\end{array}$ & 10.76 & 9.76 & 9.18 & 7.65 & 6.59 & 6.06 & 4.82 & 4.00 & 3.12 & 2.29 & 1.76 \\
\hline \hline $\begin{array}{c}\text { Brightness } \\
\text { for MF }\end{array}$ & 8.72 & 5.89 & 10.17 & 6.28 & 8.06 & 5.33 & 4.67 & 2.56 & 2.17 & 6.28 & 5.78 \\
\hline
\end{tabular}


Poirson, JASA

Table VI : Pairs for which the difference of brightness is significantly perceived as different (Friedman test with P-value indicated in the corresponding cell)

\begin{tabular}{|c|c|c|c|c|c|c|}
\hline \multirow{2}{*}{\multicolumn{2}{|c|}{$\mathrm{AM}$}} & \multicolumn{5}{|c|}{$\begin{array}{l}\text { Instrument } \\
\text { for sound } 2\end{array}$} \\
\hline & & \multirow[t]{2}{*}{ T0 } & \multirow[t]{2}{*}{$\mathrm{T} 2$} & \multirow{2}{*}{ T5 } & \multirow{2}{*}{$\begin{array}{l}\mathrm{T} 6 \\
1 \% \\
\end{array}$} & \multirow{2}{*}{$\frac{\mathrm{T} 10}{\mathrm{~T} \%}$} \\
\hline $\bar{\Xi}$ & $\mathrm{T} 0$ & & & & & \\
\hline $\begin{array}{l}\text { क } \\
\text { ț }\end{array}$ & $\mathrm{T} 2$ & & & & $2 \%$ & $1 \%$ \\
\hline$\stackrel{\overrightarrow{0}}{\stackrel{4}{0}-}$ & T5 & & & & & $1 \%$ \\
\hline 声 & $\mathrm{T} 6$ & $1 \%$ & $2 \%$ & & & \\
\hline$\Xi$ & $\mathrm{T} 10$ & $1 \%$ & $1 \%$ & $1 \%$ & & \\
\hline
\end{tabular}

\begin{tabular}{|c|c|c|c|c|c|c|}
\hline \multirow{2}{*}{\multicolumn{2}{|c|}{ MF }} & \multicolumn{5}{|c|}{$\begin{array}{l}\text { Instrument } \\
\text { for sound } 2\end{array}$} \\
\hline & & \multirow[t]{2}{*}{ T0 } & \multirow[t]{2}{*}{$\mathrm{T} 2$} & \multirow{2}{*}{ T5 } & \multirow{2}{*}{\begin{tabular}{l|} 
T6 \\
$1 \%$
\end{tabular}} & \multirow[t]{2}{*}{$\mathrm{T} 10$} \\
\hline $\bar{\Xi}$ & T0 & & & & & \\
\hline $\begin{array}{l}0 \\
\dot{0} \\
\ddot{0}\end{array}$ & $\mathrm{~T} 2$ & & & $1 \%$ & $1 \%$ & $1 \%$ \\
\hline 莺- & $\mathrm{T} 5$ & $3 \%$ & $1 \%$ & & & \\
\hline$\Xi$ & T6 & $1 \%$ & $1 \%$ & & & \\
\hline 泀 & $\mathrm{T} 10$ & & $1 \%$ & & & \\
\hline
\end{tabular}

\begin{tabular}{|c|c|c|c|c|c|c|}
\hline \multirow{2}{*}{\multicolumn{2}{|c|}{ SIMU }} & \multicolumn{5}{|c|}{$\begin{array}{l}\text { Instrument } \\
\text { for sound } 2\end{array}$} \\
\hline & & \multirow[t]{2}{*}{ T0 } & \multirow{2}{*}{ T2 } & \multirow{2}{*}{\begin{tabular}{l|} 
T5 \\
$1 \%$
\end{tabular}} & \multirow{2}{*}{$\begin{array}{ll}\mathrm{T} 6 \\
1 \% \\
\end{array}$} & \multirow{2}{*}{\begin{tabular}{|l|}
$\mathrm{T} 10$ \\
$1 \%$ \\
\end{tabular}} \\
\hline $\bar{\Xi}$ & T0 & & & & & \\
\hline 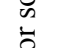 & $\mathrm{T} 2$ & & & & $1 \%$ & $1 \%$ \\
\hline$\stackrel{4}{\overrightarrow{0}}-$ & T5 & $1 \%$ & & & & $2 \%$ \\
\hline$\Xi$ & T6 & $1 \%$ & $1 \%$ & & & \\
\hline 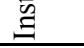 & $\mathrm{T} 10$ & $1 \%$ & $1 \%$ & $2 \%$ & & \\
\hline
\end{tabular}


Table VII : Resonance frequencies, Q-values, magnitude of the resonance peaks obtained by circle-fitting. The resonance parameters and inharmonicity corresponding to peak 4 and peak 8 of the eleven following input impedances $-\mathrm{T} 0, \mathrm{~T} 1, \mathrm{~T} 2, \ldots$ and T10 - are given

\begin{tabular}{||c||c|c|c||c|c|c||c||}
\hline \multicolumn{1}{|c||}{} & \multicolumn{3}{c||}{$4^{\text {th }}$ resonance } & \multicolumn{3}{c||}{$8^{\text {th }}$ resonance } & Inharm. \\
& $\mathrm{f}_{\text {res4} 4}(\mathrm{~Hz})$ & $\mathrm{Q}_{4}$ & $\left|\mathrm{Z}_{4}\right|$ & $\mathrm{f}_{\text {res8 }}(\mathrm{Hz})$ & $\mathrm{Q}_{8}$ & $\left|\mathrm{Z}_{8}\right|$ & $\mathrm{f}_{\text {res8 }} / 2 . f_{\text {res4 }}$ \\
\hline \hline T0 & 465.2 & 41.7 & 36.9 & 912.9 & 46.3 & 35.2 & 0.9812 \\
\hline $\mathrm{T} 1$ & 463.5 & 40 & 37.2 & 909.2 & 46.2 & 33.5 & 0.9808 \\
\hline $\mathrm{T} 2$ & 461.7 & 39.9 & 37.4 & 906.4 & 47.8 & 31.9 & 0.9816 \\
\hline T3 & 459.9 & 39.5 & 37.7 & 904 & 46.2 & 30.4 & 0.9828 \\
\hline T4 & 458 & 39 & 37.8 & 902.1 & 45.5 & 29 & 0.9848 \\
\hline T5 & 456 & 37.6 & 38 & 900.6 & 46.9 & 27.7 & 0.9875 \\
\hline T6 & 454.1 & 38.8 & 38.1 & 899.3 & 47.8 & 26.6 & 0.9902 \\
\hline T7 & 452.1 & 37.2 & 38.2 & 898.2 & 47.2 & 25.5 & 0.9934 \\
\hline T8 & 450 & 36.7 & 38.3 & 897.2 & 45.1 & 24.5 & 0.9969 \\
\hline T9 & 448 & 36.9 & 38.3 & 896.3 & 45.4 & 23.6 & 1.0003 \\
\hline T10 & 446 & 35.9 & 38.2 & 895.6 & 46.5 & 22.8 & 1.0040 \\
\hline \hline
\end{tabular}


Poirson, JASA

Table VIII : Spectral Centroid for AM, Simu and MF for each instrument

\begin{tabular}{|c|c|c|c|c|c|c|c|c|c|}
\hline \multirow[b]{2}{*}{$\mathrm{Sc}$} & \multicolumn{9}{|c|}{ Instruments } \\
\hline & T0 & \begin{tabular}{l|l}
$\mathrm{T} 1$ & $\mathrm{~T} 2$
\end{tabular} & T3 & T4 & T5 & T6 & & $\mathrm{T} 8$ & \begin{tabular}{l|l|} 
T9 & T10
\end{tabular} \\
\hline AM & 2.72 & 2.742 .65 & 2.37 & 2.46 & 2.32 & 2.38 & 2.14 & 2.18 & \begin{tabular}{|l|l|}
2.16 & 2.16 \\
\end{tabular} \\
\hline Simu & 2.62 & 2.572 .54 & 2.51 & 2.48 & 2.45 & 2.42 & 2.39 & 2.36 & \begin{tabular}{|l|l|}
2.34 .32 \\
\end{tabular} \\
\hline MF & 2.53 & 2.502 .71 & 2.55 & 2.54 & 2.52 & $\mid 2.66$ & 2.61 & 2.65 & \begin{tabular}{|l|l|}
2.77 & 2.82 \\
\end{tabular} \\
\hline
\end{tabular}


Poirson, JASA

Table IX : Correlation coefficient between the brightness score and the spectral centroid

\begin{tabular}{|c|c|c|c|}
\hline & AM & Simu & MF \\
\hline $\mathrm{R}_{\text {Pearson }}$ & 0.93 & 0.99 & -0.04 \\
\hline
\end{tabular}


Poirson, JASA

Table $\mathrm{X}$ : Correlation coefficients between the brightness score and the variables $|\mathrm{Z} 8| /|\mathrm{Z} 4|$, Q8/Q4, fres8/2.fres4

\begin{tabular}{|c|c|c|c|}
\hline & AM & Simu & MF \\
\hline $\mathrm{R}_{\text {Pearson }}\left|\mathrm{Z}_{8}\right| /\left|\mathrm{Z}_{4}\right|$ & 0.96 & 0.99 & 0.63 \\
\hline $\mathrm{R}_{\text {Pearson }} \mathrm{Q}_{8} / \mathrm{Q}_{4}$ & -0.81 & -0.85 & -0.57 \\
\hline $\mathrm{R}_{\text {Pearson }} \mathrm{f}_{\text {res8 } 8} / 2 . \mathrm{f}_{\text {res4 }}$ & -0.90 & -0.95 & -0.54 \\
\hline
\end{tabular}


Poirson, JASA

Table XI: Determination coefficient of the multiple linear regressions between the brightness scores and the factorial scores

\begin{tabular}{|c|c|c|c|}
\hline & AM & Simu & MF \\
\hline $\mathrm{R}^{2}$ & 0,89 & 0,98 & 0,37 \\
\hline
\end{tabular}


Poirson, JASA

\section{FIGURE CAPTION}

Figure 1 : Cross section of the mouthpiece with two positions of the depth : shallow(T0, left) and deep (T10, right)

Figure 2 : Measured input impedance (magnitude in $\mathrm{dB}$ and phase) of the trumpet with the "shallow" mouthpiece T0 (continuous) and with the "deep" mouthpiece T10 (dotted).

Figure $3:$ Interface for test 1

Figure 4 : Analysis of the resonances $n^{\circ} 4$ and $n^{\circ} 8$ of the impedance curve magnitude (arbitrary units)

Figure 5 : Factorial plane $|\mathrm{Z} 8| /|\mathrm{Z} 4|$ - Q8 / Q4 - fres8 / 2 fres4 

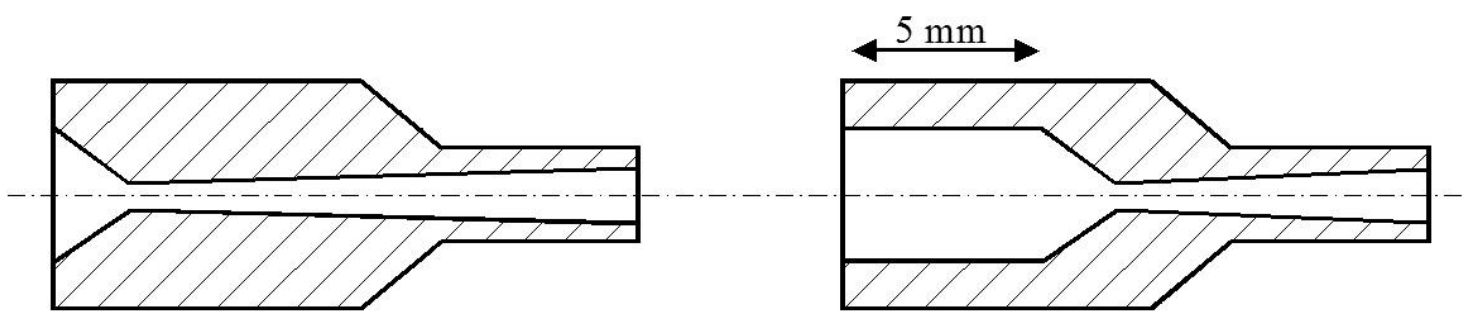

Figure 1 

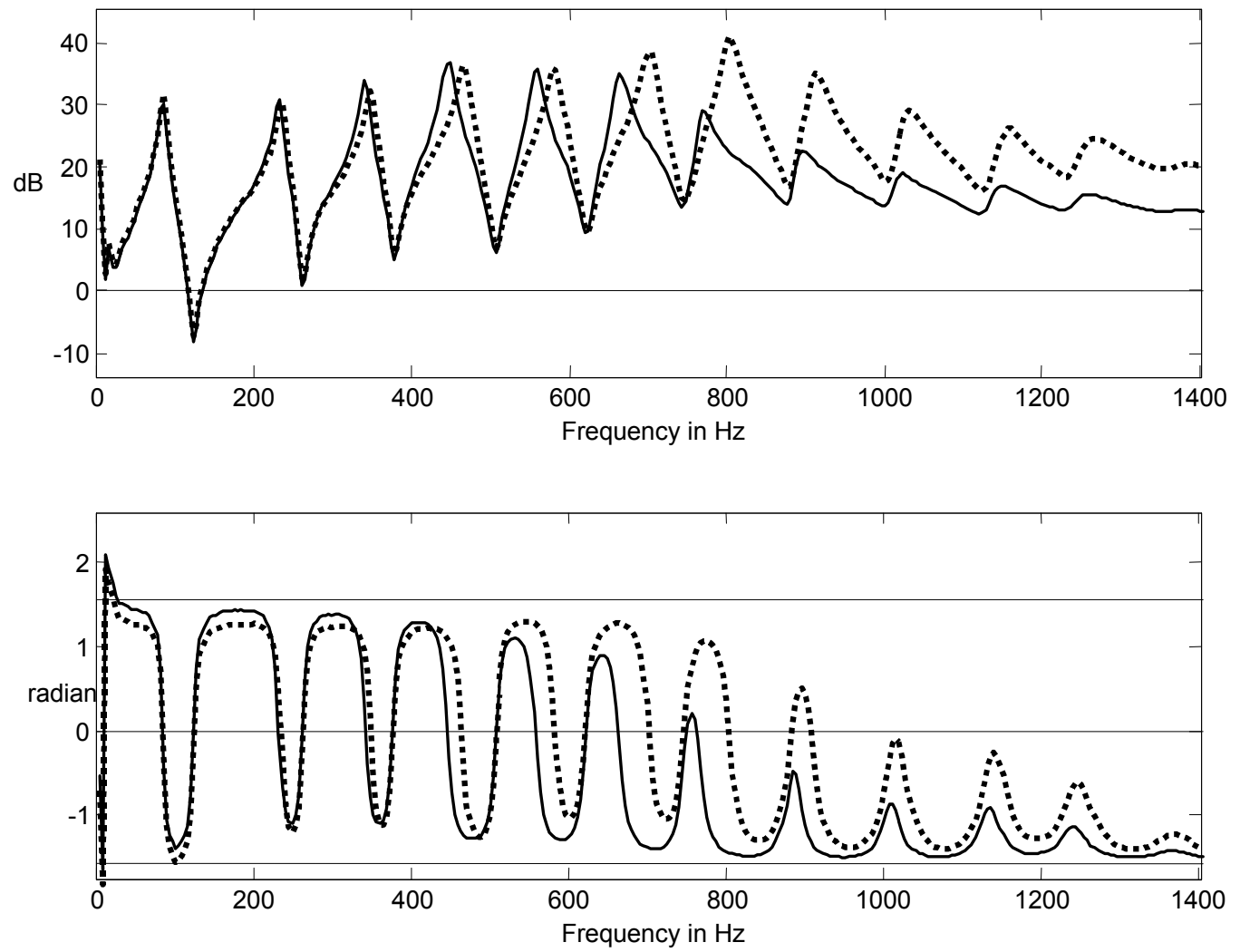

Figure 2 
Listen to the pairs of sounds and say if they are different or identical.

$18 / 99$

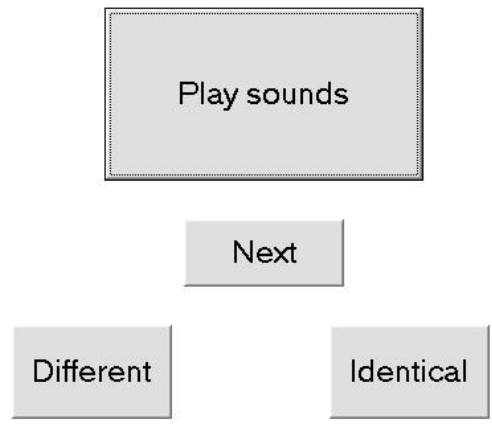

Sound 1 and sound 2 are different.

Figure 3 


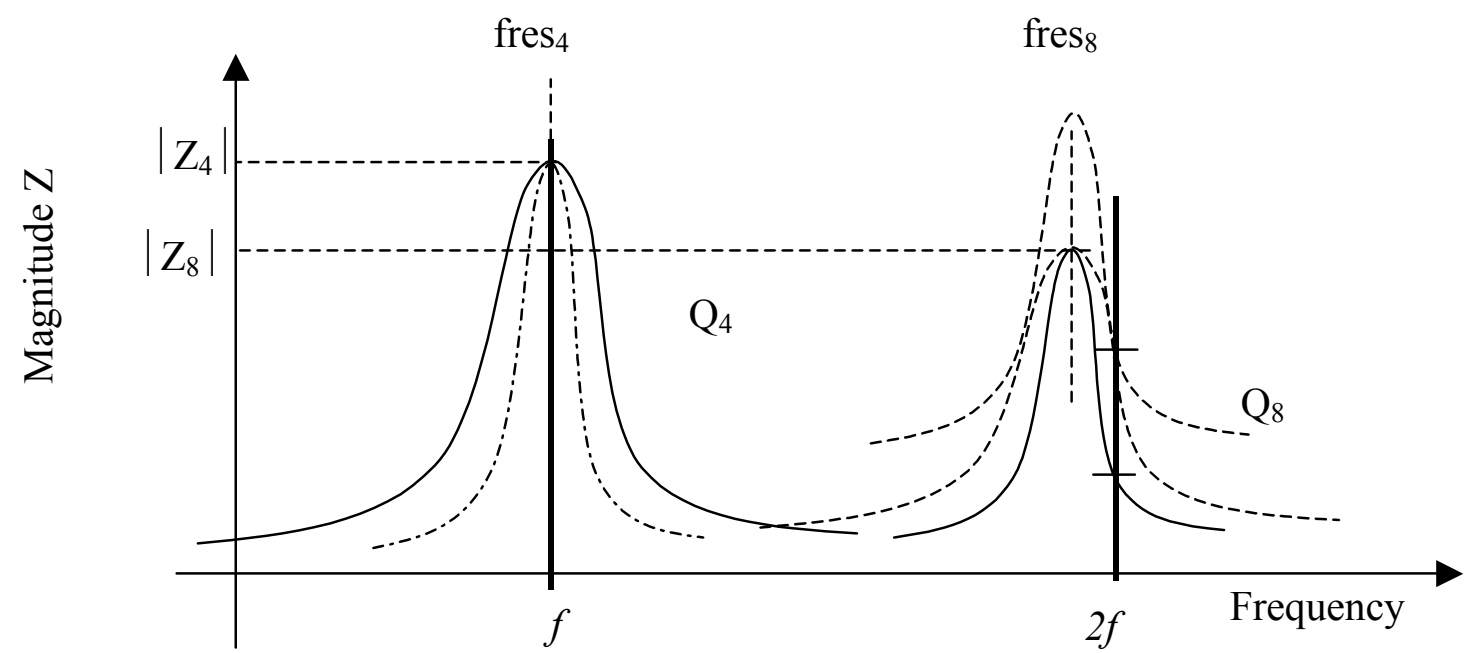

Figure 4 


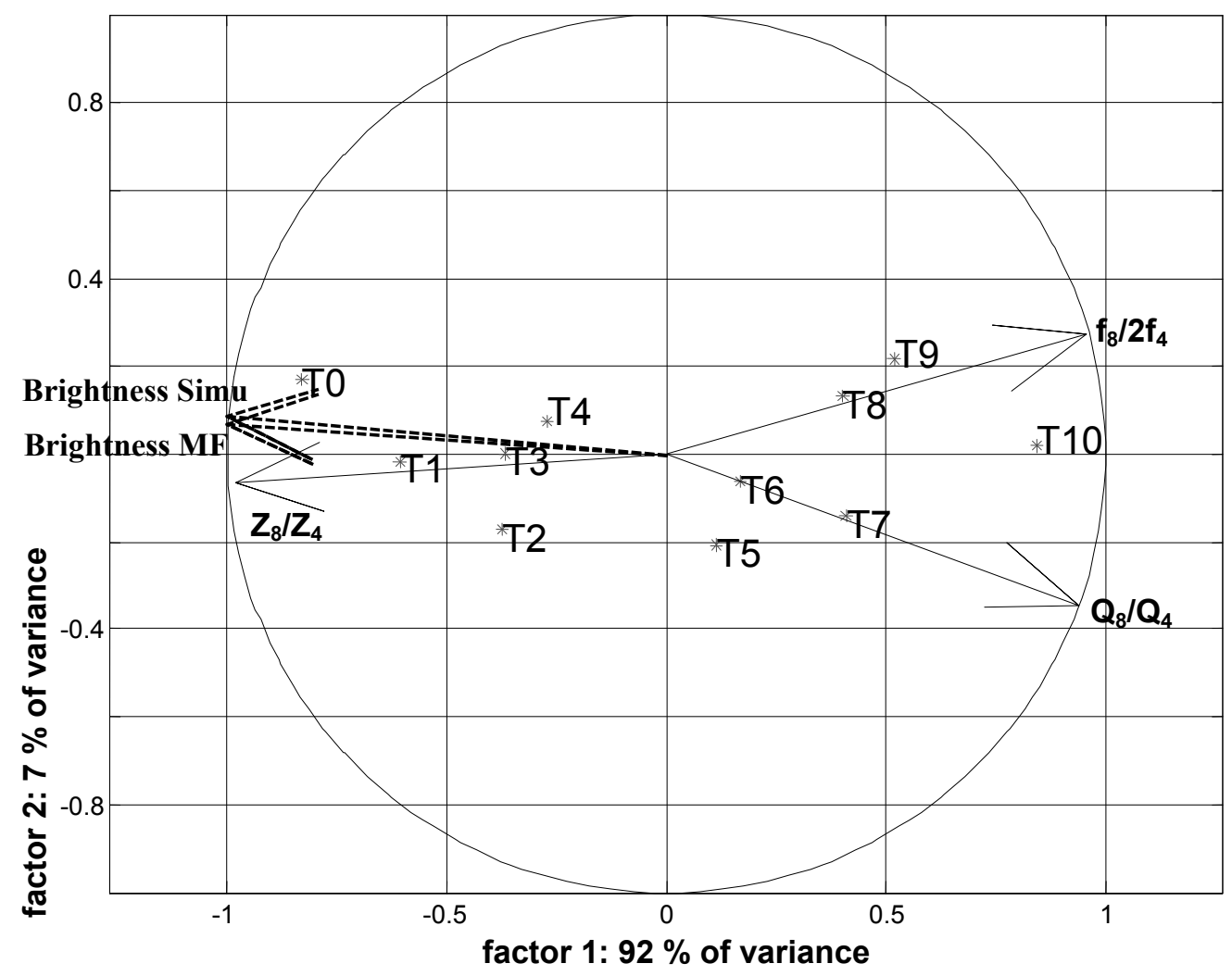

Figure 5 